\title{
BMJ Open What clinical challenges are associated with diagnosing and managing work- related mental health conditions? A qualitative study in general practice
}

\author{
Samantha Paubrey Chakraborty (D) , ${ }^{1}$ Jacinta Dermentzis, ${ }^{1}$ Bianca Brijnath, ${ }^{1,2}$ \\ Eli Ivey, ${ }^{1}$ Danielle Mazza ${ }^{1}$
}

To cite: Chakraborty SP, Dermentzis J, Brijnath B, et al. What clinical challenges are associated with diagnosing and managing work-related mental health conditions? A qualitative study in general practice. BMJ Open 2020;10:e037734. doi:10.1136/ bmjopen-2020-037734

- Prepublication history and additional material for this paper are available online. To view these files, please visit the journal online (http://dx.doi. org/10.1136/bmjopen-2020037734).

Received 18 February 2020 Revised 03 June 2020 Accepted 15 June 2020

A) Check for updates

(c) Author(s) (or their employer(s)) 2020. Re-use permitted under CC BY-NC. No commercial re-use. See rights and permissions. Published by BMJ.

${ }^{1}$ Department of General Practice, Monash University, Notting Hill, Victoria, Australia

${ }^{2}$ National Ageing Research Institute Inc, Parkville, Victoria, Australia

Correspondence to Dr Samantha Paubrey Chakraborty; samantha.chakraborty@ monash.edu

\section{ABSTRACT}

Objective When providing care for patients with work-related mental health conditions (MHCs), the general practitioner's (GP) role includes clinical care, patient advocacy and assessment of a patient's ability to work. GPs can experience difficulty representing these competing roles. As clinical guidelines were being developed to assist GPs in providing this care, our aim was to identify the clinical challenges GPs experience when diagnosing and managing patients with work-related MHCs.

Design Qualitative research.

Setting This study was conducted in general practice and workers' compensation settings across Australia.

Participants Twenty-five GPs, seven psychiatrists and nine compensation scheme workers. GPs were eligible to participate if they were actively treating (or treated within the previous three years) patient(s) who had submitted a workers' compensation claim for a MHC. Psychiatrists and compensation scheme workers were eligible to participate if they were active in these roles, as they are best placed to identify additional clinical challenges GPs themselves did not raise.

Method Participants were invited by letter to participate in qualitative semi-structured telephone interviews. Prior to each interview, participants were asked to reflect on two case vignettes, each depicting a patient's illness trajectory over 12 months. Data were thematically analysed using inductive and deductive techniques and then categorised by stages of clinical reasoning.

Results Participants reported clinical challenges across four key areas: (1) Diagnosis (identifying appropriate diagnostic tools, determining the severity and workrelatedness of a $\mathrm{MHC}$, and managing the implications of labelling the patient with MHC). (2) Management (determining optimal treatment, recommending work participation). (3) Referral (ambiguity of communication pathways within compensation schemes). (4) Procedure (difficulties navigating compensation systems).

Conclusion We found that GPs experienced clinical challenges at all stages of care for people with workrelated MHCs. We were also able to identify systemic and procedural issues that influence a GP's ability to provide care for patients with work-related MHCs.
Strengths and limitations of this study

- This study identified the clinical dilemmas faced by general practitioners (GPS) when diagnosing and managing mental health conditions that have arisen due to work

- It illuminates what topics should be included in clinical guidelines that aim to support GPs to diagnose and manage work-related mental health conditions.

- Triangulating the views of GPs, psychiatrists and compensation scheme workers strengthened the study as it enabled verification and/or explanation of the GPs' clinical dilemmas.

- A limitation of this study is that the case studies (which we used to stimulate the conversation in interviews) were limited by diversity of patient stories.

\section{INTRODUCTION}

Mental health conditions (MHCs) that have arisen as a result of work, or 'work-related MHCs', are increasing. ${ }^{1}$ These conditions may arise where work factors contribute directly to the development of a MHC or as a comorbid or secondary stressor. ${ }^{23}$ People who have an accepted claim for a work-related MHC take on average three times longer to return to work (RTW) than the median time for all claims. ${ }^{4}$ Previous studies in primary care found prevalence estimates of common mental disorders among working age people (18-65 years) ranging from $26 \%$ to $50 \% .^{6-8}$

Australian general practitioners (GPs, also known as family doctors) have a longestablished role in work capacity certification, ${ }^{9}{ }^{10}$ and are often conflicted in their dual role as patient advocates and gatekeepers to workers' compensation schemes. In Australia, $97 \%$ of injured workers seek care from a $\mathrm{GP}^{11}$ perceiving their GP as clinician, care coordinator, and navigator of the health and compensation systems. ${ }^{12}$ In their role, GPs often work with compensation schemes and 
independent medical examiners to help determine if a patient is eligible to receive workers' compensation and when a patient can return to work.

Many GPs describe challenges in enacting these roles. Our previous work revealed wide discrepancies in the amount of time off work GPs certified for work-related MHC as opposed to physical injuries, ${ }^{13}$ difficulties in assessment and diagnosis because of the invisibility of MHCs, concern that the patient may face stigma at work, and concern that the claims process itself or untimely return to work exacerbated patient's MHC.9 ${ }^{14} 15$ To address these barriers, GPs wanted clarity around certification and guidance on how to diagnose and manage work-related MHCs. ${ }^{9}{ }^{12}$ These findings are echoed internationally, ${ }^{16}$ emphasising that across primary care settings, the challenges with managing patients with work-related MHC are consistent, and that GPs internationally might benefit from the development of clinical guidelines to assist in the diagnosis and management of work-related MHCs.

Until recently, there were no clinical practice guidelines available to assist GPs in overcoming the clinical challenges with diagnosing and managing patients with work-related MHCs. To be useful in clinical practice, clinical guidance must be relevant to the end users, easy to understand and easy to implement in practice. ${ }^{17}$ However, the existing body of evidence did not identify the specific aspects of clinical care that are difficult in practice, which a guideline should explicitly address. The present study sought to determine the clinical dilemmas that GPs face when diagnosing and managing patients with work-related MHCs to inform the development of a new guideline and ensure the relevance of the guideline to GPs.

\section{METHODS}

\section{Patient and public involvement}

This research was informed by qualitative research with patients, employers and GPs who described suboptimal care for work-related MHCs. ${ }^{912}$ In this present study, we explored care delivery from the perspective of clinicians who provide the care (ie, GPs) and those who support GPs to provide this care (ie, independent medical examiners and compensation scheme workers (CSWs)), to better understand clinical challenges that resulted in suboptimal care for patients. Interviews were based on previously validated case vignettes that described the de-identified patient experiences of two patients with their GP over a period of 12 months. ${ }^{10}$

No patients were involved in the conduct of the study. However, a patient member of the project governance team was involved in analysis of the findings. The findings of this study have been disseminated to participants as a summary in the published clinical guideline. ${ }^{18}$

\section{Participants and design}

Semi-structured phone interviews were undertaken across Australia with GPs, psychiatrists (who work with compensation schemes to provide independent assessment of patients and advice regarding rehabilitation and work participation) and CSWs (who review applications for compensation claims and oversee the case management for people with accepted claims). Together, these groups are familiar with the clinical challenges experienced by GPs with regards to work-related MHCs. By including psychiatrists and CSWs in this study, we anticipated that these groups might identify additional clinical challenges that GPs themselves did not raise.

\section{Sampling}

GPs were purposively sampled by geographical location, rurality and gender and were eligible to participate if they were actively treating or had treated (within the previous threeyears) patient(s) who had submitted a workers' compensation claim for a work-related MHC. GPs were recruited from the Australasian Medical Publishing Company (AMPCo) database. The AMPCo database contains a list of approximately 29000 GPs who practise across Australia and who have consented to receive invitations to participate in research.

Psychiatrists and CSWs were also purposively sampled by geographical location. Psychiatrists were eligible to participate if they were active as independent medical examiners with a compensation scheme and CSW were eligible to participate if they were active in the role of managing claims for work-related MHCs. Psychiatrists and CSWs were recruited through the existing networks of the project team and project sponsors, which included compensation agencies in Australia.

Ethical approval for this study was provided by the Monash University Human Research Ethics Committee and participants provided consent in writing prior to the telephone interview.

\section{Procedure}

A postal invitation that explained the purpose of the study and intention to use study results to inform the development of a guideline, along with an expression of interest to participate and a consent form, were mailed to 242 GPs on the AMPCo database. Follow-up occurred by telephone at 2 weeks to non-responders. Finally, we used snowballing to enhance recruitment, whereby participating GPs were encouraged to pass the study information onto their eligible colleagues.

To recruit psychiatrists and CSWs, project team members and sponsor representatives distributed an explanatory letter and a consent form agreeing to be contacted by the research staff to psychiatrists and CSWs in their networks that met the eligibility criteria. Interested psychiatrists or CSWs returned the completed form directly to the researchers. A member of the research team then provided the potential participant with a detailed explanation of the study and sought their consent to participate. Recruitment and data analysis were conducted concurrently so that recruitment could stop when data saturation occurred. 
In line with clinical consulting rates, participating GPs and psychiatrists were reimbursed for their time with a gift voucher for $\$ A 150$, while participating CSWs, who were salaried, did not receive reimbursement. Interviews were audio recorded and professionally transcribed.

Interviews were conducted from July to September 2016. Prior to each interview, participants were given two case vignettes (see online supplementary boxes 1 and 2). The vignettes were also made available to participants at the time of the interview.

Participants used these vignettes to reflect on their own experiences of care for patients with work-related MHCs in the general practice setting. This included how GPs determine appropriate diagnostic tools, their management style, their attitude towards certifying patients and recommending RTW, and their perceived challenges and knowledge gaps.

Case vignettes and interview questions were refined following piloting with an advisory panel of GPs (clinical educators at the Department of General Practice, Monash University) for clinical accuracy and also with a recruited GP, psychiatrist and CSW. Minimal revisions were made to the written vignettes. Consequently, pilot interview data were included in the analysis.

\section{Analysis}

De-identified interview transcripts were imported into NVivo V. $11^{19}$ and thematically analysed using inductive and deductive techniques. ${ }^{20}{ }^{21}$ Two researchers conducted three iterative rounds of coding to develop the code list. After finalising the code list, the remaining transcripts were coded by a single researcher with new codes discussed between the researchers and discrepancies resolved by a third. Thereafter, codes were categorised according to the process of clinical reasoning ${ }^{22} 23$ and then clustered thematically (see table 1).

Clinical reasoning is a systematic process used by clinicians to diagnose and manage care in practice. ${ }^{22425}$ The diagnostic phase involves history taking, physical and mental examination, and investigations. The management phase includes explaining the diagnosis to the patient and providing relevant treatment, conducting procedural activities, specialist referral and monitoring progress in the patient's condition. The research team met to discuss the final interpretation of the data.

\section{RESULTS}

\section{Demographics}

Altogether, 25 GPs, seven psychiatrists and nine CSWs were interviewed. Participants were between 28 and 69 years old, and were from all states of Australia except the Australian Capital Territory. GPs were located across metropolitan and rural regions in Australia, with 16 GPs based in metropolitan Australia (eg, major capital city or other region with a population of 100000 or more) and 9 GPs based in rural Australia (in region with a population of 10000 to 100000 ), but no GPs were located in remote Australia. GPs had a median of 14 years of experience working with patients who have work-related injuries, while psychiatrists and CSWs had a median of 17 years of experience working in compensable injury.

\section{Main findings}

Interviews lasted 25-55min. GPs, psychiatrists and CSWs acknowledged the complexity of the GP role, the importance of the GP as coordinator of care and challenges with service availability. Yet all three groups identified challenges throughout the clinical reasoning pathway for patients with work-related MHCs (table 1). The three groups largely identified similar themes associated with diagnosis and management of work-related MHCs in general practice, but there was some variation in the identified challenges that were also impacted by systemic influences.

The key themes that influenced GPs' practice in relation to work-related MHCs were as follows: (a) Forming an accurate diagnosis of a MHC in relation to work; (b) How to discuss a diagnosis of a MHC with a patient; (c) Setting patient expectations for recovery and RTW; (d) Knowing when to refer the patient to other specialists; (e) Determining whether work participation could be included in the treatment approach and facilitating safe RTW; (f) Using pharmacological treatments appropriately; (g) Providing clinical care that is not hindered by the anticipation of procedural impacts on the patient; (h) monitoring and facilitating recovery. We expand on these challenges below.

\section{(a) Forming an accurate diagnosis of a MHC in relation to work} Some GPs were confident in their choice of a diagnostic tool, with preference given to DSM-5 criteria, ${ }^{26}$ and the use of the Kessler 10-item or Depression and Anxiety Stress Scales 21-item questionnaires. Others were less confident and felt guidance would be helpful:

I'd like to know what sort of depression scale would be more useful or what sort of questionnaire score that could guide the GPs as well. Saying look, if they use those scaling scores... it will give you an indication if it's above this, you know... GP12.

Psychiatrists and CSWs described concerns about inappropriate diagnostic methods and the impact of an incorrect diagnosis for the patient:

The first diagnosis will stick. And it may be only much later that we revise the diagnosis and that sometimes complicates things. P3

A consistent challenge, described by the majority of GPs, was difficulty in ascertaining the role of work in contributing to the MHC, particularly in patients where symptoms might be caused or exacerbated by non-work factors:

I think all GPs would have difficulty, if [a patient] was having some other external stressors, actually separating out, is this just work-related, is there something else going on? Has she had depression before and is this an exacerbation triggered by perhaps work? GP2. 


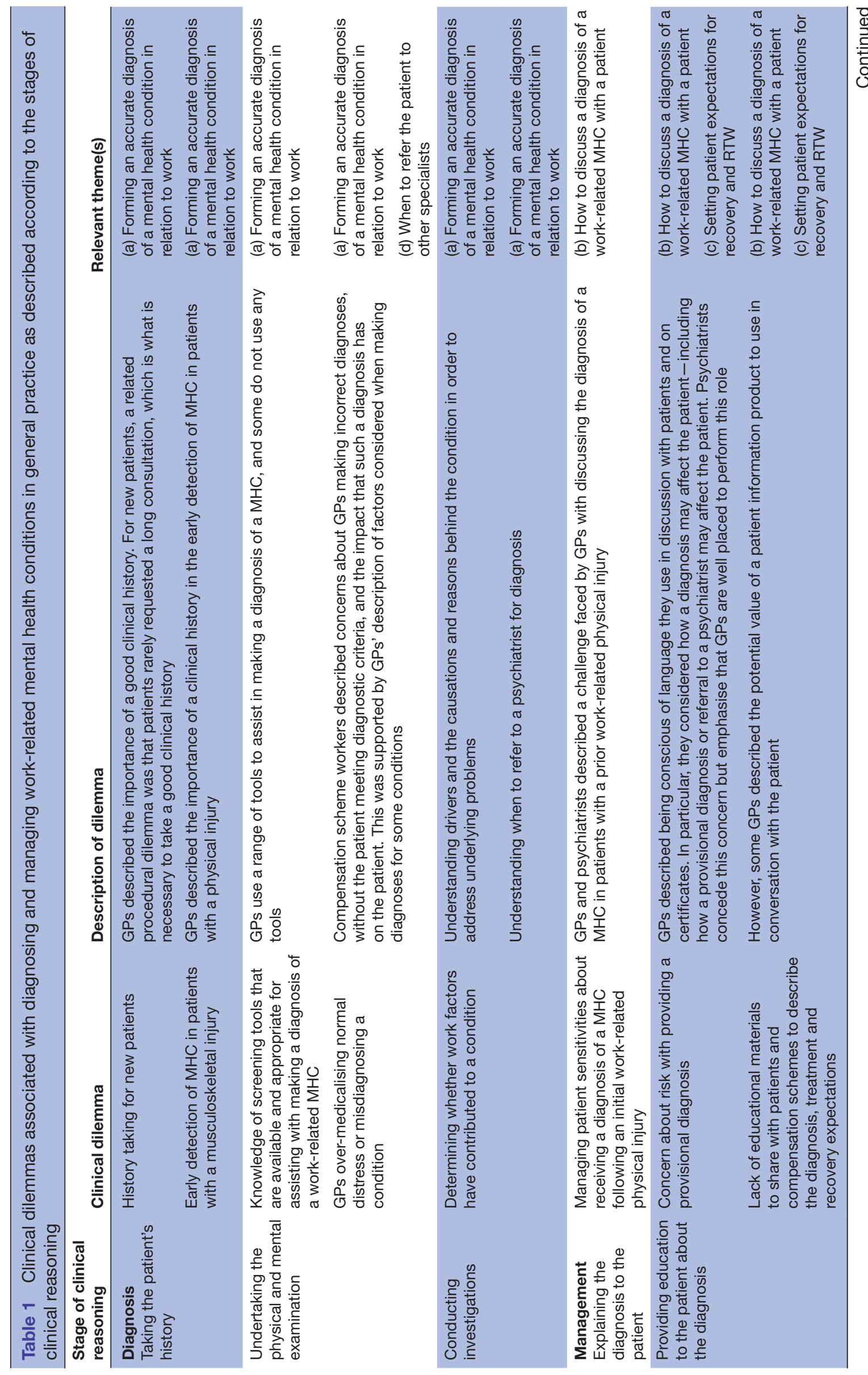




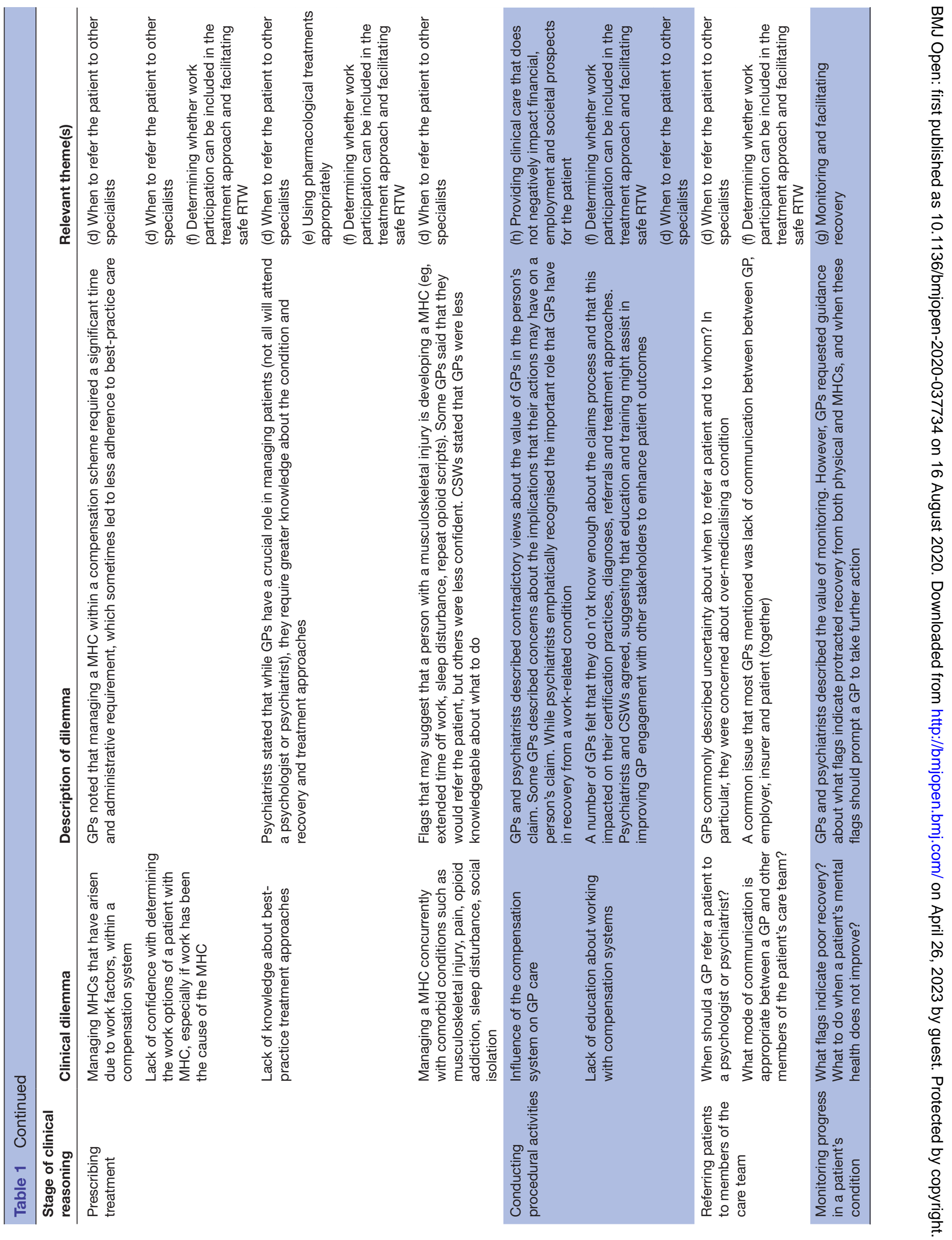


Several GPs felt that they did not know enough about the claims process, which affected their certification practices, diagnoses, referrals and treatment approaches.

How do I approach employers? Is there a format, a method, a pathway that allows me to contact the employer? Is there any obligation on the employer to discuss issues? I mean, obviously with patient's consent.... But I don't know of any pathway if there is one. GP11

Some participants noted that, in the absence of sound communication procedures with workplaces and others, GPs relied on patient reports in ascertaining whether work factors had contributed to the condition; however, they were cautious about the accuracy of this method:

If the GP uncritically accepts the patient's perspective, that can be very illness-affirming. P4

\section{(b) How to discuss a diagnosis of a MHC with a patient}

GPs were conscious of their language when discussing MHCs, treatment and recovery expectations with a patient. They were also conscious of the impact of a diagnosis and referral to a psychiatrist, noting a lack of published materials to facilitate discussions with patients. GPs perceived patient information products to use in conversation with the patient as important:

I think there could be a screening tool to assist with discussing mental health in patients who present with a physical injury... people say 'it wasn't even my fault this happened, and now my life's stuffed, and how am I ever going to have control of my life again?' GP21

\section{(c) Setting patient expectations for recovery and RTW}

The majority of participants noted that it was important to set positive RTW expectations early with the patient. However, some GPs were concerned that discussing recovery expectations, particularly RTW, could undermine their therapeutic relationship with the patient, as the patient may feel that their MHC is invalidated by their GP. Some GPs suggested that this could result in the patient doctor-shopping, or compliance issues:

Patients don't always at [12 months after a musculoskeletal injury] like that idea [of formulating a RTW plan and gradually going back], I find. I've had one or two [patients] that have actually gone to see another doctor, because I've been pushing the back to work plan too much. GP1

\section{(d) Knowing when to refer the patient to other specialists}

The majority of GPs, psychiatrists and CSWs agreed that early referral was key to ensuring a patient with a workrelated MHC was appropriately managed:

The biggest thing for me that stands out... is the early treatment, early referral. CSW9
Where a person with a musculoskeletal injury is developing a MHC, some GPs said that they would refer the patient, but CSW described GPs as being less confident:

...the GPs that I deal with and again the registrars are not quite sure what to do when they hear those flags. CS7

On the other hand, some participants expressed concern over premature referral, noting the possible negative implications for the patient:

Your patient then has a label... 'It's confirmed, I'm sick... And look, I've been referred to a psychiatrist...' The GP wouldn't have done that if he wasn't concerned about my health. CSW1

It was suggested that guidance should be included around appropriate timeframes for GPs to make referrals during diagnosis and management:

I think it would be helpful for guidelines to state how quickly to involve others in the care... or when that should take place. Because often those sorts of assessments take place a long time after the initial contact with the patient. GP1

Commonly, rural GPs noted limited availability of specialists in their community. As a result, some rural GPs suggested role-splitting with a different practitioner in their town:

If there are no other services available and you're trying to manage being the therapist as well as being the coordinator that is actually really difficult to do... I would suggest that you as the GP should make yourself the coordinator and the person who coordinates the rehabilitation, treatment and the RTW process, and that you actually get the therapy and the management of the actual problem addressed by a different practitioner. GP6

GPs, psychiatrists and CSWs described case-conferences and exchanging letters as useful methods of communication with other health professionals. GPs, however, highlighted problems finding a suitable time for the case conferences and compensation for the GP's time:

Well you can hear everybody's point of view... Everybody else can hear everybody else's point of view, and then it gets them all problem solving together.... It's a much more effective way of doing things. GP4

Across the three groups, there was consensus that GPs should continue to coordinate patient care after referral:

GPs care for people as a whole person... Generally, the role is to be the primary care provider, coordinate care including RTW. That includes diagnosis, assessment and so on... GP24

The GP as the senior medical person, the senior treating person, apart from the specialist, should be guiding things aggressively or assertively from day 
one. They should be setting the pace and they should have the confidence to do this. P1

...the GP should be the coordinator of a care team. CSW1

\section{(e) Using pharmacological treatments appropriately}

All groups described challenges related to GPs overseeing pharmacological approaches. While all three groups agreed that medication should not be used as a first-line treatment for non-severe MHCs, CSWs and psychiatrists remained cautious about GPs over-medicalising MHC and some GPs provided examples of their own non-evidencebased pharmacological use:

I think it's important that the GP doesn't medicalise on the first instance something that might not be medical. P6

I've got a basic rule of thumb that says if you're the depressive sort that is very emotional, in tears and verging on panic attacks I'll use an SSRI. If you're a depressive type that goes and locks themselves in their room or withdraws from company I'll use SNRI. Not very scientific but it seems to work. GP11

\section{(f) Determining whether work participation can be included in the treatment approach and facilitating safe RTW}

Most GPs recognised the health benefits of safe work and felt comfortable communicating this to their patients. GPs, however, described practical concerns associated with ensuring safe RTW, and that this concern led them to restrict duties:

[We]... get that people need to get back to work, and to be at work, but then I think when it comes to the practicalities of making that happen, sometimes it's easier to just give them some time off. GP21

A related procedural challenge was GPs perceived limited authority of their role when discussing RTW with other clinicians, employers, insurers and patients:

I have as a medical practitioner, I have limited power to say to someone in a company or organisation, hey you need to get this fixed. GP14

\section{(g) Monitoring and facilitating recovery}

GPs described recovery largely in terms of RTW, either at the original workplace or a different workplace.

She's not been at work for six months... I'd be really quite worried about-that's treatment failure, to me. GP5

GPs and psychiatrists also described challenges with monitoring recovery when treatment was provided by a range of health professionals.

It is said to be a major lack of specialities, particularly psychiatry, in that we do not communicate. If a GP is not getting a letter back in a timely fashion from the psychiatrist, he should be ringing that psychiatrist and saying where's my... letter? P1

(h) Providing clinical care that does not negatively impact financial, employment and societal prospects for the patient

GPs in rural locations described specific clinical challenges, and some benefits, associated with managing claims where patients and employers live in close proximity.

I've managed [a claim] where the manager is actually a good friend of mine and so all of those things in remote places, it always just complicates things a little bit more, and because usually there isn't anybody else to refer it to. GP6

Overall, GPs were concerned about the impact of their procedural activities with the compensation system on patient outcomes, which led some GPs to temper their approaches and hesitate with care decisions such as what to write on certificates, when to refer patients to members of the care team and how to monitor progress in a patient's condition. Psychiatrists, in contrast, recognised the important role that GPs have in recovery from a work-related condition.

They [GPs] are an integral part of this process. I think sometimes they may feel quite disempowered in their ability to guide and support their patients. P3

\section{DISCUSSION}

This study identified clinical dilemmas faced by GPs when diagnosing and managing patients with workrelated MHCs throughout the clinical reasoning pathway. Dilemmas were found during initial assessment and diagnosis (eg, determining which diagnostic tools are relevant, determining the severity and work-relatedness of a patient's MHC, and managing the implications of labelling the patient as having a mental health disorder), devising and actioning a management plan (including considering whether a patient can engage in work, appropriate communication with the patient's workplace, appropriate prescription of medication, and determining when and to whom referrals should be made) and monitoring a patient's recovery. In addition to clinical dilemmas, GPs described procedural difficulties that also impeded care (eg, difficulties navigating conversations with employers, understanding the compensation system and access to care from other health professionals).

\section{Comparison with existing literature}

Results expand on the previously described clinical challenges in general practice. For instance, while the clinical issue of diagnosis has been described previously, ${ }^{9} 1012$ this study demonstrated that some of these diagnostic challenges might be a result of inconsistent use of appropriate tools to assist in diagnosis and determination of the work-relatedness of a condition. Further, this 
study highlighted challenges faced by some GPs when conveying a diagnosis of a MHC to patients, including setting appropriate expectations regarding treatment and recovery with the patient. In addition, while issues around care coordination and management are described in the literature, ${ }^{12}$ this study highlighted specific challenges associated with coordinating and monitoring treatment strategies, ensuring appropriate use of medications and influencing work participation as a treatment option for patients. Finally, this study provided greater insight into the differences in clinical dilemmas faced by rural GPs compared with their metropolitan counterparts. GPs in rural and remote Australia described additional complexities relating to managing workers' compensation care in small communities and referral for psychological and workplace rehabilitation services. Furthermore, GPs in rural settings described managing patient concerns about stigma and mistrust in the community, as well as conflicts of interest where an employer might also be the GP's patient. However, close proximity was also advantageous, with rural GPs describing a good awareness of the community and the workplaces. This close relationship was considered useful for overcoming clinical challenges and is in line with similar positive experiences from occupational physicians who are engaged closely with workplaces. ${ }^{27}$

One notable inconsistency between this study and previous studies was that the results did not reveal any clinical dilemmas about alcohol or substance misuse, which are highly prevalent comorbidities for patients with MHCs. ${ }^{28}$ This may be due to the content of the two patient case studies used in the interviews, which did not discuss substance misuse in detail.

\section{Strengths and weaknesses}

Triangulating the views of GPs, psychiatrists and CSWs strengthened the study as it enabled verification and/ or explanation of the GPs' clinical dilemmas. They also help to explain some of the tensions regarding the role of the GP: for example, CSW were concerned about overdiagnosis and over-medication by GPs whereas psychiatrists regarded the GP's role in their patient's care as important. In addition, the views of these other key stakeholders enabled us to identify further dilemmas that GPs themselves did not describe, but were facing in practice. However, there was a limited range of case studies (which we used to stimulate the conversation in interviews) in both number and diversity of stories. A second limitation was that only GPs who had treated patients who submitted a claim were eligible to participate in the study (rather than GPs who had treated someone with a work-related MHC). This sampling characteristic may influence the findings of the study, as the experience of supporting a patient through a workers' compensation claim could affect the experience of these GPs. ${ }^{12}$ Finally, we should note issue of reflexivity. As this is a qualitative paper, there is a possibility that the researchers themselves may have influenced the data collection and analysis with their own previous experience of qualitative data on this topic (or personally managing work-related $\mathrm{MHCs}^{9}$ in general practice).

\section{Implications for research and practice}

This study directly informed the development of clinical guidelines for GPs on diagnosing and managing workrelated MHCs. ${ }^{18}$ By using clinical reasoning as a thematic framework to categorise these challenges, we were able to arrange these challenges according to the practical stages of a clinical consultation. This layout was applied to the presentation of topics in the guideline to create a document that aligns with the progression of clinical dilemmas that GPs are likely to face during consultations with patients. We anticipate that this user-centred approach will enhance guideline implementation, which is important given the frequently low uptake of clinical guidelines especially in general practice. ${ }^{17}$

While this study was undertaken in Australia, delivery of care for people with work-related MHCs remains a challenge internationally. ${ }^{29-31}$ Many systemic changes have been made to improve certification practices including revising sick notes to fit-notes ${ }^{16}$ and providing guidelines to implement use of revised certification. ${ }^{32}$ However, these changes have had limited effect on patient outcomes. The clinical challenges described in the present study have not, to our knowledge, been investigated internationally, yet they align with the vast and complex determinants of sickness absence that are described in the literature. ${ }^{33}$ Therefore, it is possible that GPs internationally face similar challenges to those described in this study and could benefit from guidelines developed to assist with overcoming these challenges.

Furthermore, by using the clinical reasoning framework, we were able to separate clinical issues from systemic ones so that the clinical dilemmas could be addressed in the guideline. For instance, as developers of a guideline, we were cognisant of the policy and geographical context in which GPs would be using the guideline (eg, broader factors in the compensation system such as red tape, staff turnover, independent medical examination etc). Similarly, we recommend that clinical guidelines are not the only mechanism to assist GPs in diagnosing and managing work-related MHCs. Further collaboration between researchers, GPs, patients, employers and, importantly, compensation systems should focus on making systemic improvements to assist GP to provide optimal care to these patients.

\section{CONCLUSION}

This study identified clinical dilemmas GPs face when diagnosing and managing patients with work-related MHCs. We found that GPs experienced clinical challenges at all stages of care for people with work-related MHCs. We were also able to identify systemic and procedural issues that influence a GP's ability to provide care for patients with work-related MHCs. This study directly informed 
the development of a new clinical guideline for GPs on the diagnosis and management of work-related MHCs, ${ }^{18}$ where evidence-based care recommendations were made in relation to each identified clinical challenge.

\section{Twitter Samantha Paubrey Chakraborty @SamanthaC_22}

Acknowledgements We gratefully acknowledge Dr Alex Wilde, who provided independent assistance in reviewing and editing the manuscript in preparation for publication, and Dr Vera Costa for her review of the manuscript prior to submission. We thank project funding representatives for their assistance in recruitment of compensation scheme workers and psychiatrists. Finally, we express our gratitude to the busy GPs, compensation scheme workers and psychiatrists who took the time to participate in an interview with us.

Contributors SPC led the study design, oversaw the acquisition of data, data analysis and interpretation of data, and was involved in drafting the final manuscript. JD led the data collection and was involved in data analysis. El was involved in data analysis and preparing a draft manuscript. BB and DM were involved in the study design and interpretation of data. All authors read and approved the final manuscript.

Funding This work was funded by the Australian Government Department of Jobs and Small Business and Comcare, Office of Industrial Relations-Queensland Government, State Insurance Regulatory Authority (NSW), ReturntoWorkSA, WorkCover WA.

Disclaimer The details of the two cases described in online supplementary boxes 1 and 2 are only fictional and not pertaining in the real life situations.

Competing interests DM, BB and SPC currently receive funding from the Australian Government Department of Jobs and Small Business and Comcare, Office of Industrial Relations-Queensland Government, State Insurance Regulatory Authority (NSW), ReturntoWorkSA and WorkCover WA.

Patient and public involvement Patients and/or the public were involved in the design, or conduct, or reporting, or dissemination plans of this research. Refer to the Methods section for further details.

Patient consent for publication Not required.

Ethics approval Low-risk approval for this study was obtained by the Monash University Human Research Ethics Committee (MUHREC no. CF16/203520162001022).

Provenance and peer review Not commissioned; externally peer reviewed.

Data availability statement De-identified participant data are available on reasonable request from the corresponding author.

Open access This is an open access article distributed in accordance with the Creative Commons Attribution Non Commercial (CC BY-NC 4.0) license, which permits others to distribute, remix, adapt, build upon this work non-commercially, and license their derivative works on different terms, provided the original work is properly cited, appropriate credit is given, any changes made indicated, and the use is non-commercial. See: http://creativecommons.org/licenses/by-nc/4.0/.

ORCID iD

Samantha Paubrey Chakraborty http://orcid.org/0000-0002-9708-4532

\section{REFERENCES}

1 Safe Work Australia. The cost of work-related injury and illness for Australian employers, workers, and the community, 2008-2009. Canberra, Australia: Safe Work Australia, 2012.

2 Carnide N, Franche R-L, Hogg-Johnson S, et al. Course of depressive symptoms following a workplace injury: a 12-month follow-up update. J Occup Rehabil 2016;26:204-15.

3 WHO. Identification and control of work-related diseases. Report of a WHO expert committee WHO Technical Report series 714 WHO. Geneva, 1985.

4 Safe Work Australia. Work-related mental disorders profile 2015. Canberra, Australia: Safe Work Australia, 2015.

5 Rivière M, Plancke L, Leroyer A, et al. Prevalence of work-related common psychiatric disorders in primary care: the French Héraclès study. Psychiatry Res 2018;259:579-86.

6 Ansseau M, Dierick M, Buntinkx F, et al. High prevalence of mental disorders in primary care. J Affect Disord 2004;78:49-55.
7 Toft T, Fink P, Oernboel E, et al. Mental disorders in primary care: prevalence and co-morbidity among disorders. results from the functional illness in primary care (FIP) study. Psychol Med 2005;35:1175-84.

8 Alkhadhari S, Alsabbrri AO, Mohammad IHA, et al. Prevalence of psychiatric morbidity in the primary health clinic Attendees in Kuwait. J Affect Disord 2016;195:15-20.

9 Brijnath B, Mazza D, Singh N, et al. Mental health claims management and return to work: qualitative insights from Melbourne, Australia. J Occup Rehabil 2014;24:766-76.

10 Brijnath B, Singh N, Mazza D. Stakeholder perspectives on the new sickness certificate in Victoria: results from a mixed-methods qualitative study. Aust Health Rev 2016;40:27-32.

11 Mazza D, Brijnath B, O'Hare MA, et al. Do health service use and return-to-work outcomes differ with GPs' injured-worker caseload? J Occup Rehabil 2019;29:64-71.

12 Mazza D, Brijnath B, Singh N, et al. General practitioners and sickness certification for injury in Australia. BMC Fam Pract 2015;16:1471-2296.

13 Collie A, Ruseckaite R, Brijnath B, et al. Sickness certification of workers compensation claimants by general practitioners in Victoria, 2003-2010. Med J Aust 2013;199:480-3.

14 Bunzli S, Singh N, Mazza D, et al. Fear of (re)injury and return to work following compensable injury: qualitative insights from key stakeholders in Victoria, Australia. BMC Public Health 2017;17:313.

15 Kosny A, Brijnath B, Singh N, et al. Uncomfortable bedfellows: employer perspectives on general practitioners' role in the return-towork process. Pol Prac Health Safety 2015;13:65-76.

16 Dorrington S, Roberts E, Mykletun A, et al. Systematic review of fit note use for workers in the UK. Occup Environ Med 2018;75:530-9.

17 Francke AL, Smit MC, de Veer AJE, et al. Factors influencing the implementation of clinical guidelines for health care professionals: a systematic meta-review. BMC Med Inform Decis Mak 2008;8:38.

18 Mazza D, Chakraborty SP, Brijnath B, et al. Diagnosing and managing work-related mental health conditions in general practice: new Australian clinical practice guidelines. Med J Aust 2019;211:76-81.

19 QSR International. NVivo qualitative data analysis software; version 10, 2014.

20 Olszewski B, Macey D, Lindstrom L. The practical work of $<$ Coding >: an ethnomethodological inquiry. J Philosophy Social Sci 2006;29:363-80.

21 Braun V, Clarke V. Using thematic analysis in psychology. Qual Res Psychol 2006;3:77-101.

22 Murtagh J. John Murtagh's general practice. 6th edn. Australia: Mcgraw Hill, 2015.

23 Kassirer JP. Teaching clinical reasoning: case-based and coached. Acad Med 2010;85:1118-24.

24 Ilgen JS, Eva KW, Regehr G. What's in a label? Is diagnosis the start or the end of clinical reasoning? J Gen Intern Med 2016;31:435-7.

25 Linn A, Khaw $\mathrm{C}$, Kildea $\mathrm{H}$, et al. Clinical reasoning - a guide to improving teaching and practice. Aust Fam Physician 2012;41:18-20.

26 American Psychiatric Association. Diagnostic and statistical manual of mental disorders. 5th edn. Arlington, VA: American Psychiatric Association, 2013.

27 Kinnunen-Amoroso M, Liira J. Work-related stress management between workplace and occupational health care. Work 2016;54:507-15.

28 Kingston REF, Marel C, Mills KL. A systematic review of the prevalence of comorbid mental health disorders in people presenting for substance use treatment in Australia. Drug Alcohol Rev 2017;36:527-39.

29 Boettcher N, Mitchell J, Lashewicz B, et al. Men's work-related stress and mental health: illustrating the workings of masculine role norms. Am J Mens Health 2019;13:155798831983841.

30 Khan A, Teoh KR, Islam S, et al. Psychosocial work characteristics, burnout, psychological morbidity symptoms and early retirement intentions: a cross-sectional study of NHS consultants in the UK. BMJ Open 2018;8:e018720.

31 Müller G, Brendel B, Freude G, et al. Work-related determinants of burnout in a nationally representative sample of German employees: results from the study on mental health at work. J Occup Environ Med 2018;60:584-8.

32 Gustavsson C, Hinas E, Ljungquist T, et al. General practitioners' use of sickness certification guidelines in Sweden at introduction and four years later: a survey study. Int $J$ Qual Health Care 2018;30:429-36.

33 de Vries H, Fishta A, Weikert B, et al. Determinants of sickness absence and return to work among employees with common mental disorders: a scoping review. J Occup Rehabil 2018;28:393-417. 